\title{
Potentiel et limites de la «médecine individuali- sée» (personalized medicine)
}

Académie Suisse des Sciences Médicales (ASSM)

\section{Le projet de cette feuille de route a été rédigé par le Prof. Andreas Papassotiropoulos de la Division des neuro- sciences moléculaires de l’Université de Bâle. Le comité de direction de l'ASSM a discuté en détail et remanié ce document lors de sa séance des 3 et 4 septembre 2012 et l'a approuvé ensuite dans sa présente forme.}

Correspondance: Académie Suisse des Sciences Médicales (ASSM) Petersplatz 13 CH-4051 Bâle

mail[at]samw.ch

\begin{abstract}
Résumé
La médecine individualisée (MI) (souvent désignée par médecine personnalisée) ouvre des perspectives nouvelles pour la compréhension de la genèse et de l'évolution des maladies; elle offre en même temps des nouvelles bases pour le développement de substances actives. Si la MI a connu un essor considérable ces dernières années, elle $\mathrm{a}$, en même temps, accentué un certain nombre de problèmes; ceux-ci sont mentionnés dans cette feuille de route qui esquisse également des solutions possibles. Actuellement, la MI est principalement appliquée dans les domaines suivants: la prédiction; le diagnostic; la thérapie (y compris l'évaluation des outcomes); le développement de nouveaux médicaments. Le domaine de la prédiction (évaluation des risques de survenue de la maladie) révèle aussi bien le potentiel que les limites de la MI. Lorsqu'il s'agit de maladies monogéniques, la MI peut fournir de bonnes prédictions. Mais la plupart des maladies sont dues à l'association de plusieurs gènes ainsi que de l'interaction de ces gènes avec des facteurs environnementaux. Dans le cas des maladies oligogéniques (qui associent environ dix gènes), il est parfois possible d'établir une prédiction partielle, alors qu'elle est impossible en cas de maladies polygéniques; dès lors, l'utilité des tests génétiques est limitée. Dans l'ensemble, une anamnèse familiale est plus informative que des prédictions issues d'analyses génétiques à large échelle.
\end{abstract}

Au niveau du diagnostic, la MI joue d'ores et déjà un rôle important. En particulier, dans le domaine de l'oncologie, les différents types de cancers sont de plus en plus souvent diagnostiqués selon leur «empreinte génétique». Egalement dans d'autres domaines, comme par exemple la cardiologie, la MI est devenue, pour de nombreux médecins, un nouvel instrument de diagnostic.

La $\mathrm{Ml}$ a permis des progrès significatifs également dans la thérapie. De plus en plus souvent, de nouvelles substances actives sont développées pour des groupes de patients aux caractéristiques moléculaires spécifiques. Cette tendance se confirme surtout en oncologie. Ainsi, non seulement l'efficacité des substances actives est améliorée pour les patients concernés, mais les effets secondaires sont diminués.

Dans le domaine du développement de nouveaux médicaments, aucune entreprise pharmaceutique ne peut se permettre aujourd'hui de négliger les données de la MI. Cellesci sont prises en compte dans toutes les étapes du développement et de la planification des études cliniques.

Mais ces progrès fulgurants soulèvent divers problèmes qui exigent dès aujourd'hui que des mesures soient adoptées. Sont cités dans la feuille de route: les lacunes des connaissances, la prolifération d'experts, les offres fantaisistes, la transparence, le consentement éclairé, la protection des données, la preuve de l'efficacité des nouvelles thérapies, les brevets et la liberté dans la recherche.

Face à ces problèmes, la présente feuille de route propose quelques solutions:

\section{Combler les lacunes des connaissances}

La MI n'en est qu'à ses débuts, mais le développement des connaissances est fulgurant. Ainsi, pour être en mesure d'utiliser correctement les résultats de la $\mathrm{Ml}$, il est important que les médecins disposent de connaissances suffisantes dans les domaines de l'épidémiologie, de la génétique et de la statistique médicale. De plus, les patients qui s'informent en ligne attendent des médecins qu'ils soient à même de répondre précisément à leurs questions.

\section{Renforcer l'importance de la génétique médicale}

La génétique fait partie intégrante de la MI. Les exigences envers les médecins sont de plus en plus élevées quant à leur capacité en matière d'analyse et de conseil génétiques. II est donc indispensable de renforcer la place de la génétique médicale dans la formation médicale.

\section{Ouvrir des domaines spéciaux}

Chaque maladie a sa propre génétique et sa complexité spécifique. C'est pourquoi il est nécessaire de promouvoir les offres de formation postgraduée et continue pour offrir à chaque médecin la possibilité d'acquérir les connaissances nécessaires à la $\mathrm{MI}$ dans son domaine. Les patients ont, eux aussi, le droit de savoir quel médecin dispose des connaissances leur permettant de répondre aux questions qui relèvent de la MI.

\section{Renforcer l'importance de l'anamnèse familiale}

Les étudiants doivent être sensibilisés à l'importance de I'anamnèse familiale pendant la formation pré- et postgraduée. Il est également indispensable d'élaborer des directives cliniques pour l'interprétation de l'anamnèse familiale et de transmettre de façon optimale les connaissances et compétences nécessaires.

\section{Eviter les dérives et exiger la transparence}

Les offres de MI librement accessibles doivent faire l'objet d'une validation scientifique afin que chaque utilisateur potentiel puisse décider en connaissance de cause. Ceci implique aussi la transparence concernant les possibles conflits d'intérêts de quelque nature qu'ils soient. II est important que les sciences médicales prennent clairement position sur les aspects qui ne peuvent pas être réglementés.

La MI est une occasion exceptionnelle pour améliorer la prédiction, la thérapie et le suivi des patients. Des connaissances solides basées sur les preuves, une pratique sérieuse ainsi que sur la définition de priorités permettront de profiter au maximum de cette innovation et d'éviter les dérives potentielles. Pour les besoins des patients, il est essentiel que les techniques de relevé et de traitement des données de la MI ne supplantent pas la relation personnelle entre le médecin et son patient, mais qu'elles y soient intégrées. 


\section{Contexte}

De tout temps, les progrès de la médecine et les conquêtes technologiques ont été étroitement liés. Qu'il s'agisse du développement de nouvelles méthodes de coloration histologique, de l'utilisation d'ondes électromagnétiques ou de la fabrication de nouveaux alliages de métaux - pour ne citer que quelques exemples -, la technologie a constamment ouvert de nouvelles perspectives à la médecine.

Depuis plus de dix ans, nous vivons, dans le domaine biomédical, une nouvelle révolution qui est basée sur deux évolutions parallèles. D'une part, on dispose d'analyses moléculaires à grande échelle, de plus en plus rapides et à haute définition qui deviennent aussi de moins en moins coûteuses (par exemple les analyses génomiques du polymorphisme, l'analyse génomique des séquences $\mathrm{ADN}$ et $\mathrm{ARN}$, l'épigénomique, la protéomique, la métabolomique). D'autre part, l'évolution des méthodes analytiques intégratives dans le domaine de la bioinformatique permet une interprétation pertinente de l'énorme quantité de données.

Ainsi s'ouvrent des perspectives inédites facilitant la compréhension des processus physiologiques et pathophysiologiques, de la genèse et du déroulement des maladies et de l'action thérapeutique. Ces nouvelles informations et connaissances sont rapidement mises en ligne sur Internet et accessibles à tous sans être filtrées. Les avancées technologiques complexes, leur application dans les sciences médicales ainsi que l'accessibilité à toutes les informations vont modifier notre manière d'appréhender la santé et la maladie et donc la médecine dans son ensemble. Ces profonds bouleversements soulèveront inéluctablement de nombreuses questions scientifiques, éthiques et politiques. Il est donc temps - également pour la Suisse - d'engager une réflexion sur ces évolutions et sur les questionnements qu'elles soulèvent. A cet égard, la science médicale a le devoir d'encourager une formation d'opinion basée sur l'évidence et aux fondements éthiques solides.

\section{Définition}

Les progrès mentionnés en introduction dans les technologies dites «omics» et les possibilités d'obtenir et d'interpréter à partir d'un individu une multitude d'informations médicales - avec des séquences géniques complètes - ont mené à une utilisation plus fréquente de l'expression «médecine personnalisée». Les termes de médecine «individualisée», «sur mesure», «stratifiée» ou «de précision» sont également utilisés. L'expression «médecine personnalisée» étant ambiguë, dans la mesure où la procédure ainsi décrite vise moins les caractéristiques spécifiques à une personne, que les structures et processus biologiques individuels, nous emploierons dans cette feuille de route le terme de «médecine individualisée» (MI).
Dans ce document, on entend par MI une approche médicale dans laquelle les caractéristiques d'un individu sont définies au niveau moléculaire et évaluées au moyen de la technologie d'information en vue d'établir un pronostic, un conseil et/ou une thérapie individualisés pour ce patient précis.

Selon la nature des données relevées, cette définition peut être interprétée étroitement (par exemple en la limitant aux données génomiques, on parle alors de «médecine génomique») ou largement en y intégrant d'autres données (par exemple des données anamnestiques ou relatives à la maladie, c'est la «médecine basée sur les données»).

Cependant, la MI ne mérite le nom de «médecine» que si le processus est envisagé déjà avant le début du relevé des données et au-delà de l'établissement d'un pronostic ou d'une recommandation. Pour que la MI tienne compte de la personnalité du patient ou de l'utilisateur, les données brutes sont insuffisantes; la relation personnelle entre le patient et le professionnel respectivement le médecin est indispensable. L'envoi d'un échantillon biologique (éventuellement accompagné d'un questionnaire rempli) et la réception d'une recommandation écrite ne sont pas (encore) de la MI.

La croissance rapide de l'usage des notions susmentionnées suggère qu'il pourrait s'agir d'un concept innovateur en médecine. Bien que la méthode dont se sert la MI soit effectivement innovatrice, l'idée sous-jacente est aussi vieille que la médecine elle-même. La «médecine individualisée» (MI) vise l'optimisation de la prise en charge médicale pour chaque individu, depuis la prévention jusqu'à la thérapie [1], en répertoriant et analysant aussi bien les particularités biologiques individuelles et les facteurs environnementaux que les déterminants agissant sur toute la durée de vie d'un individu. En réalité, cet objectif de la MI correspond à la nature de la médecine depuis Hippocrate au moins. La concentration avec l'unicité de l'individu est la caractéristique de toute activité médicale correcte. Les bases de données de la littérature médicale contemporaine montrent que l'utilisation de la notion de «médecine personnalisée» connaît depuis le début de ce siècle une croissance exponentielle. Néanmoins, dès les années 1970 et 1990, quelques rapports isolés traitaient de ce thème. Ces publications prévoyaient même un déclin de la MI, c'est-à-dire de la focalisation sur l'individu et du conseil individuel, en arguant de la technicisation croissante et de l'«algorithmisation» de la médecine $[2,3]$. Ces doutes confirment que l'être humain est plus que la somme des données relevées sur sa personne. Conformément à sa nature, il ne peut être appréhendé que dans ses échanges avec une autre personne. Dès lors, il est essentiel que les techniques de relevé et de traitement des données de la MI ne supplantent pas la relation personnelle entre le médecin et son patient, mais qu'elles y soient intégrées. 


\section{Domaines d'application de la «médecine individualisée»}

Comme exposé ci-dessus, le concept de la MI n'est ni nouveau, ni exceptionnel; bien au contraire, il représente un des fondements de la pensée médicale. Cependant la médecine est arrivée à un point où les évolutions technologiques et analytiques élargissent considérablement l'éventail des possibilités préventives, diagnostiques et thérapeutiques et bouleversent quelquefois notre compréhension des processus à l'œuvre. De ce fait, dans l'immédiat, il est utile de définir les domaines d'application de la MI moderne. Les progrès les plus rapides, les plus complets et les plus concrets pour la pratique étant actuellement réalisés dans la génomique, ce domaine sert souvent de référence. Mais dès à présent, les domaines de l'expression génétique, de l'épigénomique, de la protéomique et de la métabolomique enregistrent des avancées similaires. Toutefois, ceux-ci n'influenceront probablement ni les domaines d'application, ni les ambitions de la MI.

Actuellement quatre domaines d'application de la MI peuvent être définis:

1. la prédiction, 2. le diagnostic, 3. la thérapie (y compris l'évaluation des outcomes), 4. le développement de nouveaux médicaments.

\subsection{La prédiction}

On entend par prédiction l'évaluation des risques et le diagnostic présymptomatiques (également prénatal), dans le but d'établir un pronostic, de poser un diagnostic et, le cas échéant, d'élaborer une stratégie préventive ou curative, à un stade précoce de la pathologie [4]. Dans des cas isolés, la prédiction génomique fait d'ores et déjà partie de notre système de prise en charge médicale, par exemple dans le cas de la chorée de Huntington. Une prédiction génomique d'une particularité génétique dominante a des conséquences non seulement pour la personne, mais aussi pour toute sa famille; dès lors, même si l'étendue du conseil, tel que l'exige la loi, est considérable, il est juste et nécessaire. Le but de cette feuille de route n'est pas de présenter en détail la dimension éthique d'une prédiction basée sur les génomes. Néanmoins, et d'autant plus à cause de la multitude d'offres, il est important de prendre conscience desdits tests prédictifs, si toutefois une prédiction basée sur les génomes est justifiable et réalisable du point de vue scientifique.

En principe, toute prédiction (y compris génomique) s'exprime sous la forme d'une probabilité. La transmission de probabilités est toutefois une entreprise complexe $[5,6]$.

La qualité d'une prédiction découle de l'amplitude d'effets du prédicteur. La MI peut donc apporter un soutien significatif en cas de maladies monogéniques qui révèlent par définition une amplitude d'effets d'une variation génétique élevée. En cas de maladies oligogéniques, caractérisées par un nombre faible de variations génétiques (rarement plus de 10) (certains types de cancer, par exemple), une prédiction juste est extrêmement difficile. D'une part, les facteurs environnementaux jouent un rôle important dans les maladies oligogéniques et, d'autre part, les variants associés à la maladie peuvent interagir entre eux, ce qui complique ou rend impossible une évaluation prédictive sérieuse. Cependant, grâce à l'épidémiologie génétique combinée à des méthodes d'analyses génomiques, l'amplitude des effets et les interactions entre les gènes peuvent être modélisées et évaluées dans le sens d'une prédiction, tout en sachant que, comparées aux maladies monogéniques, la qualité des prédictions est largement inférieure (par exemple [7]).

Cette problématique est accentuée pour les maladies polygéniques (par exemple maladie coronarienne, hypertension artérielle, démences, maladies psychiatriques). Dans la plupart des cas, le nombre élevé de facteurs de risque génétiques et non génétiques, leurs interactions compliquées, empêchent une prédiction fiable au niveau individuel. En fait, lorsqu'il s'agit d'établir un pronostic individuel, un facteur de risque génétique significatif, identifié au moyen d'analyses statistiques de groupes (par exemple l'allèle e4 de l'apolipoprotéine dans la maladie d'Alzheimer), peut être d'aucune utilité $[8,9]$. Il faut également souligner que chaque maladie comporte sa propre génétique et sa complexité spécifique. La génétique de l'hypertension artérielle est différente de celle du carcinome pancréatique, et cette dernière est différente de celle du trouble affectif bipolaire, même si toutes ces maladies sont réunies sous le terme de maladies polygéniques.

Ces dernières années, des études d'association génomiques à haute définition (genome-wide association studies, GWAS) ont examiné des millions de polymorphismes génétiques dans de très grands collectifs (parfois avec plus de 100000 participants). Elles ont permis d'identifier une multitude de facteurs de risque génétiques dupliqués et des gènes de susceptibilité pour différentes maladies polygéniques. Ces études représentent un énorme progrès dans la compréhension de la physiopathologie des maladies en question et ont déjà permis de développer des nouvelles approches thérapeutiques [10].

Mais lorsqu'il s'agit d'évaluer le risque individuel de maladie au moyen d'un variant identifié - une promesse propagée à des fins publicitaires par certaines entreprises -, les résultats anéantissent toute illusion. Malgré les nombreuses études génétiques réalisées à large échelle, les variants identifiés ne remplissent pas les critères de validation des tests prédictifs. Compte tenu de la complexité des maladies polygéniques, ce résultat n'est pas surprenant. Toutefois, il était intéressant de constater à plusieurs reprises, que chaque effet "prédictif» de la variante de risque génétique, aussi significatif soit-il, devient 
négligeable (du point de vue statistique) lorsque l'anamnèse familiale est intégrée dans le modèle (par exemple [11]).

En d'autres termes: dans le cas de l'évaluation du risque individuel de maladie polygénique, une anamnèse familiale approfondie prime largement sur n'importe quel test génétique basé sur les variants associés au risque. Une anamnèse familiale approfondie tient toujours compte de l'ensemble de tous les facteurs de risque génétiques ou non (mais partagés) au sein de la famille ainsi que le modèle complexe d'interaction de ces facteurs; elle est bien plus parlante que n'importe quelle analyse génétique qui, aussi large soit-elle, reste finalement isolée. L'anamnèse familiale représente pour chaque praticien un instrument très efficace pour une évaluation fondée et sérieuse des risques dans le sens de la MI. Malheureusement, l'anamnèse (qu'elle soit familiale ou personnelle) est souvent négligée dans la pratique médicale quotidienne et est mal intégrée dans la prise en charge médicale [12].

\subsection{Le diagnostic}

L'apport de la MI dans le diagnostic et le pronostic des maladies est d'ores et déjà significatif et son potentiel de développement est élevé. L'oncologie, qui a recours à des méthodes modernes basées sur la MI pour parvenir à un diagnostic moléculaire et à une caractérisation moléculaire des tumeurs malignes, fait figure de précurseur dans ce domaine. Le domaine de l'oncologie présente l'avantage de pouvoir réaliser directement les examens génomiques, épigénomiques et protéomiques sur le tissu tumoral et permettre une sous-typisation détaillée de chaque maladie. Qu'il s'agisse de cancers du sein, du colon, de lymphomes, ou de leucémies, etc., la MI a permis un développement considérable des connaissances, dont profitent directement les patients. Plusieurs génomes complets de cancer ont déjà pu être séquencés, comme par exemple ceux de la leucémie myéloïde aiguë et de certains carcinomes bronchiques [13-15]. Il existe de grands consortiums internationaux qui se consacrent à la caractérisation complète de tissus tumoraux - de l'ADN jusqu'à la protéine -, ainsi par exemple pour le glioblastome et le cancer du sein.

En plus de l'oncologie, d'autres domaines spécialisés utilisent la MI pour élargir le répertoire diagnostique et pronostique, comme par exemple la cardiologie. Au moyen d'approches métabolomiques et protéomiques, on examine s'il est possible de différencier les différents stades d'un infarctus du myocarde aigu et d'une angine de poitrine instable [16, 17]. Egalement divers tests basés sur l'expression génétique dans le sang sont validés pour l'évaluation du degré de gravité d'une maladie coronarienne [18, 19]. En résumé, on peut sans aucun doute insister sur l'importance de la MI pour améliorer le diagnostic et le pronostic de nombreuses maladies en faveur des patients.

\subsection{La thérapie (y compris l'évaluation des outcomes)}

Dans le domaine de la thérapie, la MI est très en avance. L'oncologie y joue également un rôle de précurseur, notamment dans la prise en compte des paramètres pharmacologiques. Il va de soi que, dans le domaine de l'oncologie, les développements de la thérapie et de l'évaluation des outcomes vont de pair avec les développements au niveau du diagnostic, c'est-à-dire d'une meilleure sous-typisation basée sur les caractéristiques moléculaires de la tumeur. Ainsi, de plus en plus des substances qui agissent uniquement chez des sous-groupes moléculaires bien définis, sont autorisées pour traiter différents types de cancer [4]. A cet égard, il existe des programmes à large échelle (par exemple en France, aux Etats-Unis, en Norvège et en Grande-Bretagne) dont le but est d'aboutir à des thérapies à l'efficacité individuelle, à l'aide d'une caractérisation moléculaire complète des biopsies tumorales. Non seulement ces développements sont pertinents en terme d'efficacité, mais ils permettent également d'éviter les traitements inutiles et potentiellement dangereux et d'évaluer les résultats thérapeutiques. Ces développements ne profitent pas seulement au domaine de l'oncologie, mais en principe à tous les domaines cliniques, comme par exemple les maladies cardiovasculaires, métaboliques, neuropsychiatriques ou dermatologiques. Appliquée à bon escient, la MI contribuera à l'amélioration significative des options thérapeutiques dans tous les domaines de la médecine.

\subsection{Le développement de nouveaux médicaments} Egalement pour le développement de nouveaux médicaments, l'application de méthodes pertinentes pour la MI revêt une importance majeure. A chaque stade du développement des médicaments (target identification, target validation, lead development, preclinical phases, clinical phases, market), le rôle de la génomique, de l'épigénomique et de la protéomique est essentiel [1]. Il est, de plus, évident qu'avant l'introduction d'un nouveau médicament, son efficacité sélective doit être vérifiée à l'aide de méthodes basées sur la MI. Si les études cliniques nécessaires sont encore plus coûteuses (surtout les études de phase III), d'un autre côté, une stratification des sujets d'étude basée sur la MI améliore l'amplitude d'effets, de telle façon que le nombre de participants à recruter pour l'étude peut être diminué. Manifestement, l'influence de la MI sur le développement des médicaments est positive et significative et va en faveur des patients. En règle générale, chaque entreprise pharmaceutique tient compte aujourd'hui de la MI dans le développement de nouveaux médicaments pour tous les domaines de la médecine clinique.

\section{Domaines problématiques}

Comme tout progrès technologique, la MI soulève de nombreuses questions qu'il importe de discuter à un 
stade précoce afin de prévenir les dérives possibles. Les huit domaines problématiques suivants méritent une attention particulière.

\subsection{Les lacunes des connaissances}

La signification et les conclusions de la MI ne peuvent être ni comprises, ni correctement interprétées sans connaissances statistiques et épidémiologiques. Les probabilités doivent toujours être comprises dans leur contexte respectif. Une probabilité de 80 pour cent de réagir à un médicament précis ou une probabilité de 80 pour cent de contracter une maladie incurable ont des significations très différentes pour l'individu concerné. La signification statistique d'un gène de susceptibilité n'exprime encore rien quant à sa valeur prédictive. Pourtant un allèle à risque avec un risque relatif de 3, par exemple, peut être insignifiant pour la prédiction de la pathologie en question. Il est étonnant de constater que le débat autour de la MI dissimule chez certaines personnes concernées des lacunes importantes concernant des principes simples d'épidémiologie et de statistique. Il importe de combler aussi rapidement et systématiquement que possible ces lacunes ainsi que d'adopter des mesures concrètes pour mieux prendre en compte les informations qu'apporte l'anamnèse familiale.

\subsection{La prolifération d'experts}

La MI englobe un domaine de connaissances très vaste, comprenant des aspects médicaux, biologiques, biotechnologiques, informatiques et éthiques. En conséquence, le discours académique autour de la MI est essentiellement pluridisciplinaire, ce qui est positif et souhaitable. Toutefois, le sens et l'objectif de la MI ne doivent pas être oubliés: la MI vise l'optimisation de la prise en charge médicale pour chaque individu, depuis la prévention jusqu'à la thérapie. La voix des experts pouvant être influente dans ce domaine très important pour la médecine, il est temps de réaliser que l'expertise auto-proclamée peut s'avérer potentiellement nuisible et que les experts en MI doivent satisfaire à des exigences minimales.

\subsection{Les offres fantaisistes}

«Si vous avez été allaitée, vous êtes porteuse d'une variante génétique qui augmente votre intelligence.» De telles offres de tests "prédictifs» sont à même de discréditer l'ensemble de la MI. Des fournisseurs internationaux connus propagent de tels tests génétiques «direct-to-consumer» (DTC), au même titre que des tests génétiques supposés prédire une bonne ou une mauvaise capacité de mémoire, un risque de schizophrénie ou une tendance suicidaire. Ces «offres» sont dépourvues de tout fondement scientifique. Elles dévoilent un sérieux problème: un marché difficile à contrôler et à réglementer s'est établi dans le domaine de la MI. En Suisse, les tests DTC sont interdits sans recommandation médicale. Mais ces interdits sont souvent inutiles, car facilement contournés dans un univers globalisé. Il est donc important de soumettre ces offres à une vérification scientifique accessible à tous, de telle façon que chaque utilisateur potentiel puisse prendre ses décisions en connaissance de cause. Des groupes de spécialistes nationaux et internationaux ont d'ores et déjà adopté une position claire et mettent en garde contre de telles offres. A cet égard, l'aide-mémoire concernant les tests génétiques disponibles sur internet, publié par la Commission d'experts pour l'analyse génétique humaine (GUMEK) est exemplaire (www.bag.admin.ch/themen/medizin/ 00683/02724/04638/07332/index.html?lang=fr).

Outre le caractère non scientifique de certaines offres, les développements dans le domaine des tests génétiques DTC soulèvent un autre problème: l'absence de conseil génétique (cf. à ce sujet 4.5.). Certaines offres DTC sont valables, comme par exemple la définition de mutations en cas de caractéristiques héréditaires dominantes ou récessives (par exemple chorée de Huntington). Toutefois, les résultats ne sont pas interprétés et les utilisateurs ne bénéficient pas des informations et des conseils nécessaires. La banalisation de cette réalité, sous couvert du droit à la liberté de rechercher des informations - droit qui n'est pas à remettre en question -, relève de la négligence. Bien plus, du point de vue de l'éthique, c'est une omission inacceptable, car il ne s'agit pas ici d'une quelconque information, mais de faits médicaux complexes qui exigent une interprétation prudente.

\subsection{La transparence}

Si l'intérêt de la MI est considérable d'un point de vue scientifique, il ne l'est pas moins du point de vue économique. S'agissant d'une orientation futuriste de la médecine, le potentiel économique est significatif. C'est justement pour cette raison, mais aussi parce qu'il s'agit de la santé de chacun, qu'une transparence totale doit être de mise. Les experts, les discutants influents, les fournisseurs de prestations de MI, les initiateurs de plates-formes de discussions publiques et de forums en ligne - tous doivent justifier leur intérêt personnel et déclarer leurs éventuelles interdépendances économiques. Si, pour un domaine aussi sensible, la transparence est une contrainte évidente, son application se doit d'être rigoureuse.

\subsection{Consentement éclairé}

S'il est prévu de relever, en une seule étape et sans indication médicale précise, un nombre élevé de données potentiellement importantes pour la santé, l'exigence d'une information complète et compréhensible sur le bénéfice et les risques de ces mesures doit être considérée sous un jour nouveau. Il va de soi que les tests génétiques exigent d'ores et déjà une information préalable sur l'éventualité de résultats inattendus et dont la signification n'est pas claire; mais jusqu'alors, comparée à l'objectif même de l'examen, ces résultats n'ont toujours retenu qu'une moindre attention. Compte tenu de la multitude de 
données potentiellement importantes que recèlent ces nouvelles possibilités d'examen, il est difficile de donner une information complète, ne serait-ce que du point de vue quantitatif. La signification d'une multitude d'informations n'est souvent pas solide et peut se transformer rapidement, il est encore donc plus difficile de donner une information adéquate. Les exigences posées à un consentement éclairé valable, dans le cadre de la MI, doivent faire l'objet d'une analyse éthique et juridique minutieuse.

Lorsque des personnes incapables de discernement sont examinées, notamment des enfants ou des nouveau-nés, ou lors d'examens prénataux ou de tests de paternité, ce problème est encore plus difficile à résoudre.

\subsection{Protection des données}

Les informations issues de la MI sont par définition très personnelles et donc sensibles. C'est pourquoi elles doivent être bien protégées. Le personnel - pas seulement médical - impliqué n'est pas toujours conscient de cette responsabilité. Les utilisateurs d'Internet doivent être expressément mis en garde contre les dangers de l'utilisation de données (propres ou concernant des tiers) aussi sensibles.

\subsection{Preuve de l'efficacité des nouvelles thérapies} Lors de la vérification de l'efficacité des nouveaux médicaments, la MI permet une stratification des sujets d'expérimentation en différents sous-groupes. Lorsqu'une substance semble inefficace pour l'ensemble de la population, mais semble très efficace pour un groupe de la population, il faut vérifier dans quelle mesure l'éfficacité est prouvée pour ce petit sous-groupe de la population. La possibilité d'exiger pour de tels sous-groupes un statut de «maladie orpheline» doit être liée à une vérification minutieuse de l'efficacité clinique et du profil adéquat des effets secondaires.

\subsection{Brevets, liberté thérapeutique et liberté de recherche}

On pourrait imaginer que le fabricant d'une substance fasse breveter un paquet constitué d'un relevé de données diagnostiques déterminées, d'un algorithme s'y rapportant et d'une prescription individualisée sur cette base. Ceci pourrait, d'une part, limiter la liberté thérapeutique du médecin traitant en l'empêchant, par exemple, de traiter d'autres facteurs ignorés par l'algorithme; d'autre part, et avant tout, un tel brevet pourrait entraver la recherche pour l'amélioration de la thérapie individualisée de cette maladie.

\section{Solutions possibles}

Les mesures proposées ci-après résultent des problèmes susmentionnés et ne doivent pas être comprises comme exhaustives. La MI n'en est qu'à ses débuts; on peut penser qu'avec le temps, d'autres points de discussion seront soulevés, qui exigeront une évaluation correspondante. Néanmoins, il est essentiel d'agir dès aujourd'hui.

\subsection{Combler les lacunes de savoir}

Les connaissances des médecins dans le domaine de l'épidémiologie, de la génétique et de la statistique médicales doivent être améliorées. De nombreuses questions de la MI présentent aussi des aspects statistiques et épidémiologiques. Il faut également être conscient qu'à l'ère de l'informatique, la plupart des patients consultent Internet et attendent de leur médecin traitant des explications, des interprétations et des réponses. Le manque de connaissances appropriées provoque des incertitudes et des peurs.

\subsection{Renforcer l'importance de la génétique médicale}

L'analyse et l'interprétation des séquences génomiques constituent une partie importante de la MI. Les exigences des patients en matière de conseil génétique envers leurs médecins sont en constante augmentation. Il est donc indispensable de mieux intégrer la génétique médicale et de renforcer son importance dans la formation médicale.

\subsection{Ouvrir des domaines spéciaux}

La MI est une nouvelle spécialisation respectivement sous-spécialisation. Elle se différencie clairement en fonction de la spécialité médicale. Comme mentionné ci-dessus, chaque maladie a sa propre génétique et sa propre complexité. Dès aujourd'hui, il est clair qu'un savoir génétique général ne suffit plus à satisfaire l'analyse de la complexité génétique de chaque maladie polygénique, respectivement à suivre le rythme des découvertes scientifiques. D'un autre côté, chaque médecin de famille, cardiologue, chirurgien, psychiatre, dermatologue, etc. sera confronté prochainement aux questions des patients concernant la MI dans son domaine respectif. Dès lors, il est essentiel de développer les offres de formation postgraduée et continue pour que chaque médecin ait la possibilité d'acquérir le savoir nécessaire à la MI dans son domaine, afin d'être en mesure de conseiller les patients, sans toutefois être obligés de suivre une formation postgraduée complète en génétique médicale. Les patients ont, eux aussi, le droit de savoir quel médecin dispose réellement du savoir nécessaire pour qu'ils soient à même de répondre aux questions qui relèvent de la MI. Finalement, le savoir nécessaire à la MI et son actualisation continuelle deviendront partie intégrante de chaque spécialité médicale.

\subsection{Renforcer l'importance de l'anamnèse familiale}

Pour ce faire, les étudiants doivent être sensibilisés à l'importance de l'anamnèse familiale pendant la formation pré- et postgraduée; il est également indispensable d'élaborer des directives cliniques pour l'in- 
terprétation de l'anamnèse familiale et de transmettre de façon optimale les connaissances et compétences nécessaires.

\subsection{Eviter les dérives et exiger la transparence}

Comme expliqué en détail ci-dessus, si certaines évolutions ne peuvent pas être réglementées, elles n'en nécessitent pas moins des positions claires du côté des sciences médicales. Celles-ci doivent être exprimées d'une manière forte et compréhensible. Les offres de MI doivent faire l'objet d'une vérification scientifique afin que chaque utilisateur potentiel puisse agir en connaissance de cause. A cet égard, les sciences médicales sont particulièrement sollicitées. Ceci implique aussi la transparence concernant les possibles conflits d'intérêts de quelque nature qu'ils soient.

\section{Perspectives}

La MI transforme continuellement notre façon d'appréhender la médecine. Elle constitue une occasion exceptionnelle pour améliorer la prédiction, la thérapie et le suivi des patients. Seules des connaissances scientifiques solides et basées sur les preuves, une pratique sérieuse ainsi que la définition de priorités dès la formation prégraduée, postgraduée et continue, permettront de tirer le maximum de cette occasion, au bénéfice de l'être humain, et d'éviter les dérives potentielles.

Bien que la MI soit exercée en priorité au niveau médical individuel, il importe aussi de prendre conscience de son importance pour la médecine et la santé publique. D'une part, des approches scientifiques intéressantes peuvent résulter du relevé d'un nombre important de données et, d'autre part, les conséquences d'une multitude de recommandations individuelles sur le comportement de la population doivent être minutieusement analysées. Les conséquences d'un besoin croissant de conseils et les questions éthiques liées à la MI devraient également faire l'objet d'une analyse approfondie.

\section{Références}

1 Chan IS, Ginsburg GS. Personalized medicine: progress and promise. Annu Rev Genomics Hum Genet. 2011;12:217:44

2 Gibson WM. Can personalized medicine survive? Can Fam Physician. 1971;17:29-88.

3 Arnold RM, Forrow L. Rewarding medicine: good doctors and good behavior. Ann Intern Med. 1990;113:794-8.
4 Meyer UA. Personalized medicine: a personal view. Clin Pharmacol Ther. 2012;91:373-5

5 Hoffrage U, et al. Medicine. Communicating statistical information. Science. 2000;290:2261-2.

6 Kurzenhauser S, Hertwig R. How to foster citizens' statistical reasoning: implications for genetic counseling. Community Genet. 2006;9:197-203.

7 Grassmann F, et al. Modelling the genetic risk in age-related macular degeneration. PLoS One. 2012;7:e37979.

8 Relkin NR, et al. The National Institute on Aging/ Alzheimer's Association recommendations on the application of apolipoprotein E genotyping to Alzheimer's disease. Ann N Y Acad Sci. 1996;802:149-76.

9 Seshadri S, et al. Genomewide analysis of genetic loci associated with Alzheimer disease. JAMA. 2010;303: $1832-40$.

10 Hirschhorn JN, Gajdos ZK. Genome-wide association studies: results from the first few years and potential implications for clinical medicine. Annu Rev Med. 2011;62:11-24.

11 Ripatti S, et al. A multilocus genetic risk score for coronary heart disease: case-control and prospective cohort analyses. Lancet. 2010;376: 1393-400.

12 Guttmacher AE, et al. The family history-more important than ever. N Engl J Med. 2004;351:2333-6.

13 Lee $\mathrm{W}$, et al. The mutation spectrum revealed by paired genome sequences from a lung cancer patient. Nature. 2010;465:473-7.

14 Ley TJ, et al. DNA sequencing of a cytogenetically normal acute myeloid leukaemia genome. Nature. 2008;456:66-72.

15 Pleasance ED, et al. A small-cell lung cancer genome with complex signatures of tobacco exposure. Nature. 2010;463:184-90.

16 Mateos-Caceres PJ, et al. Proteomic analysis of plasma from patients during an acute coronary syndrome. J Am Coll Cardiol. 2004;44:1578-83.

17 Sabatine MS, et al. Metabolomic identification of novel biomarkers of myocardial ischemia. Circulation. 2005;112:3868-75.

18 Rosenberg S, et al. Multicenter validation of the diagnostic accuracy of a blood-based gene expression test for assessing obstructive coronary artery disease in nondiabetic patients. Ann Intern Med. 2010;153:425-34

19 Wingrove JA, et al. Correlation of peripheral-blood gene expression with the extent of coronary artery stenosis. Circ Cardiovasc Genet. 2008;1:31-8. 